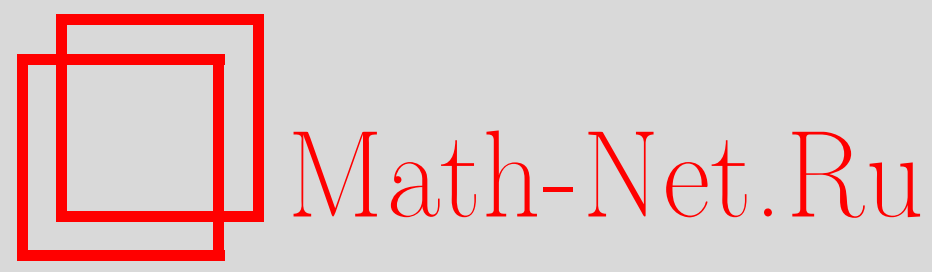

М. И. Тихомирова, В. П. Чистяков, Об оценке периодов зашумленных двоичных периодических последовательностей, Дискрет. матем., 2009, том 21, выпуск 3, 24-32

DOI: https://doi.org/10.4213/dm1058

Использование Общероссийского математического портала Math-Net.Ru подразумевает, что вы прочитали и согласны с пользовательским соглашением http://www . mathnet.ru/rus/agreement

Параметры загрузки:

IP: 35.174 .16 .151

26 апреля 2023 г., 13:22:13 


\title{
Об оценке периодов зашумленных двоичных периодических последовательностей
}

\author{
() 2009 г. М. И. Тихомирова, В. П. Чистяков
}

\begin{abstract}
Для оценки длин периодов зашумленных двоичных последовательностей предлагаются и исследуются статистики, равные логарифму функции правдоподобия, и близкие к ним.
\end{abstract}

\section{1. Введение}

При проверке качества последовательностей псевдослучайных чисел $\left\{x_{t}^{*}\right\}$ за основную гипотезу $H_{0}$ принимается предположение, по которому исследуемая последовательность $\left\{x_{t}^{*}\right\}$ является реализацией последовательности независимых равномерно распределенных случайных величин $\left\{x_{t}\right\}$. Во множество предполагаемых конкурирующих гипотез включается обычно предположение о периодичности (в том или ином смысле) последовательности $\left\{x_{t}\right\}$.

Одним из возможных подходов к проверке предполагаемой периодичности является подход, основанный на использовании дискретного преобразования Фурье (см. [1, 2]). Пусть

$$
x_{1}, x_{2}, \ldots, x_{t}, \ldots
$$

- случайные величины, принимающие значения 0 и 1 . Положим

$$
Y_{j}=\frac{T}{2}\left|X\left(\omega_{j}\right)\right|^{2}, \quad j=1, \ldots, L, \quad L=[T / 2],
$$

где

$$
X(\omega)=\frac{2}{T} \sum_{t=1}^{T}\left(2 x_{t}-1\right) e^{i \omega t}, \quad \omega_{j}=\frac{2 \pi j}{T} .
$$

По гипотезе $H_{0}$ случайные величины (1) независимы и вероятности каждого из знаков 0 и 1 равны $1 / 2$. Для проверки гипотезы $H_{0}$ используем статистику $Y_{j_{0}}=\max _{j} Y_{j}$. Если гипотеза $H_{0}$ неверна, то в качестве оценок частоты $\omega_{0}$ и соответствующего ей истинного периода $n_{0}$ принимаются $\widehat{\omega}=\omega_{j_{0}}$ и $\hat{n}_{0}=T / j_{0}$.

В нашей работе исследуются двоичные последовательности (1), которые по конкурирующей гипотезе $H_{1}$ являются зашумленными случайными периодическими последовательностями, определяемыми следующим образом. Пусть $\left\{z_{t}\right\},\left\{y_{t}\right\},\left\{\varepsilon_{t}\right\}-$ случайные двоичные последовательности и

$$
\mathbf{P}\left\{z_{t}=1\right\}=\mathbf{P}\left\{y_{t}=1\right\}=1 / 2, \quad \mathbf{P}\left\{\varepsilon_{t}=1\right\}=p, \quad 0<p<1, \quad t=1,2, \ldots
$$


Случайные величины $y_{1}, \ldots, y_{n_{0}}, z_{t}, \varepsilon_{t}, t=1,2, \ldots$, независимы. Последовательность $\left\{y_{t}\right\}$ является периодической:

$$
\mathbf{P}\left\{y_{n_{0} l+m}=y_{m}, l=0,1, \ldots, m=1,2, \ldots, n_{0}\right\}=1 .
$$

Зашумленная периодическая последовательность $\left\{x_{t}\right\}$ определяется следующим образом:

$$
x_{t}=\varepsilon_{t} y_{t}+\left(1-\varepsilon_{t}\right) z_{t}, \quad t=1,2, \ldots
$$

В работе получено явное выражение для функции правдоподобия выборки, состоящей из $T$ первых членов последовательности (1), предложены статистики, которые могут быть использованы для оценки периода последовательности, исследуются свойства этих статистик.

\section{2. Функция правдоподобия}

Напомним, что $n_{0}-$ период последовательности $\left\{y_{t}\right\}$. Найдем распределение первых $T=n_{0} L_{0}, L_{0}>1$, членов последовательности $\left\{x_{t}\right\}$. Положим $x(T)=\left(x_{1}, \ldots, x_{T}\right)$, $y\left(n_{0}\right)=\left(y_{1}, \ldots, y_{n_{0}}\right)$. Возможные значения этих векторов обозначим через $g(T)$ и $h\left(n_{0}\right)$ :

$$
g(T)=\left(g_{1}, \ldots, g_{T}\right), \quad h\left(n_{0}\right)=\left(h_{1}, \ldots, h_{n_{0}}\right), \quad g_{t}, h_{t} \in\{0,1\} .
$$

Теорема 1. Совместное распределение $T=n_{0} L_{0}$ первых членов последовательности $\left\{x_{t}\right\}$, определенной равенством (4), имеет следующий вид:

$$
\mathbf{P}\{x(T)=g(T)\}=\frac{1}{2}\left(\frac{1-p}{2}\right)^{n_{0} L_{0}} e^{G_{n_{0}}(g(T))},
$$

гेe

$$
\begin{aligned}
G_{n_{0}}(T) & =\sum_{m=1}^{n_{0}} \ln \left(A^{\chi_{m}\left(n_{0}\right)}+A^{L_{0}-\chi_{m}\left(n_{0}\right)}\right), & & A=\frac{1+p}{1-p}, \\
\chi_{m}\left(n_{0}\right) & =\sum_{l=0}^{L_{0}-1} \chi\left(g_{n_{0} l+m}=1\right), & & m=1, \ldots, n_{0},
\end{aligned}
$$

$\chi(C)-$ индикатор события $C$.

Доказательство. Используя равенство (4) при $t=n_{0} l+m$, получим, что

$$
\begin{aligned}
\mathbf{P}\left\{x(T)=g(T) \mid y\left(n_{0}\right)=h\left(n_{0}\right)\right\} & =\prod_{l=0}^{L_{0}-1} \prod_{m=l}^{n_{0}} \mathbf{P}\left\{\varepsilon_{n_{0} l+m} h_{m}+\left(1-\varepsilon_{n_{0} l+m}\right) z_{n_{0} l+m}=g_{n_{0} l+m}\right\} \\
& =\prod_{l=0}^{L_{0}-1} \prod_{m=l}^{n_{0}}\left(p \chi\left(h_{m}=g_{n_{0} l+m}\right)+(1-p) / 2\right) .
\end{aligned}
$$

Отсюда и из равенств

$$
1+\frac{2 p}{1-p} \chi\left(h_{m}=g_{n_{0} l+m}\right)=\left(1+\frac{2 p}{1-p}\right)^{\chi\left(h_{m}=g_{n_{0} l+m}\right)}, \quad \mathbf{P}\left\{y\left(n_{0}\right)=h\left(n_{0}\right)\right\}=\frac{1}{2^{n_{0}}}
$$


следует, что

$$
\mathbf{P}\left\{x(T)=g(T), y\left(n_{0}\right)=h\left(n_{0}\right)\right\}=\frac{(1-p)^{n_{0} L_{0}}}{2^{n_{0}\left(L_{0}+1\right)}} A^{\sum_{m=1}^{n_{0}} \sum_{l=0}^{L_{0}-1} \chi\left(h_{m}=g_{n_{0} l+m}\right)} .
$$

Суммируя обе стороны равенства (6) по всем значениям $h\left(n_{0}\right)$, получим, что

$$
\mathbf{P}\{x(T)=g(T)\}=\frac{(1-p)^{n_{0} L_{0}}}{2^{n_{0}\left(L_{0}+1\right)}} \sum_{h_{1}, \ldots, h_{n_{0}}} A^{\sum_{m=1}^{n_{0}} \sum_{l=0}^{L_{0}-1} \chi\left(h_{m}=g_{n_{0} l+m}\right)} .
$$

Отсюда следует утверждение теоремы.

Если период $n_{0}$ неизвестен, то для оценки периода последовательности $\left\{y_{t}\right\}$ по последовательности $\left\{x_{t}\right\}$ можно использовать статистики

$$
\xi_{n}(T)=\sum_{m=1}^{n} \ln \left(A^{v_{m}^{(T)}(n)}+A^{L_{n}-v_{m}^{(T)}(n)}\right), \quad n=2,3, \ldots,
$$

где $L=[T / n]$, а частоты $v_{m}^{(T)}(n)$ определяются равенствами

$$
v_{m}^{(T)}(n)=\sum_{l=0}^{L_{n}-1} x_{n l+m}
$$

Будет показано, что при больших $T$ распределение статистики $\xi_{n_{0}}(T)$ заметно отличается от распределений статистики $\xi_{n}(T)$ с $n$, не кратным $n_{0}$.

\section{3. Моменты и распределения частот}

Будем считать, что неизвестный период $n_{0}$ последовательность $\left\{y_{t}\right\}$ является одним из $r+1$ натуральных чисел заданного множества. Числа этого множества, отличные от $n_{0}$, обозначим через $n_{1}, \ldots, n_{r}$. Частоты (8) удобно записать в виде

$$
v_{m}^{(T)}\left(n_{i}\right)=\sum_{t \in Z_{m}\left(n_{i}\right)} x_{t}, \quad i=0,1, \ldots, r
$$

где

$$
Z_{m}\left(n_{i}\right)=\left\{t: t=n_{i} l+m, l=0,1, \ldots,\left[T / n_{i}\right]-1\right\}, \quad m=1,2, \ldots, n_{i} .
$$

Множества (10), соответствующие истинному периоду, обозначим $M_{m}=Z_{m}\left(n_{0}\right)$. Для упрощения формул ковариаций частот (9) ограничимся рассмотрением случая, когда

$$
T=n_{0} n_{1} \ldots n_{r} L
$$

где $L-$ целое число, $L \geqslant 1$, числа $n_{i}, i=0,1, \ldots, r$, являются попарно взаимно простыми. Число элементов множества $C$ будем обозначать через $|C|$.

При условии (11)

$$
\left|Z_{m}\left(n_{i}\right)\right|=\frac{T}{n_{i}}, \quad\left|Z_{m_{1}}\left(n_{i}\right) \cap Z_{m_{2}}\left(n_{j}\right)\right|=\frac{T}{n_{i} n_{j}}, \quad i \neq j .
$$


Математические ожидания, дисперсии, ковариации и распределения вероятностей при гипотезах $H_{0}$ и $H_{1}$ будем обозначать $\mathbf{E}_{l}, \mathbf{D}_{l}, \operatorname{cov}_{l}, \mathbf{P}_{l}, l=0,1$, соответственно.

При гипотезе $H_{0}$

$$
\mathbf{E}_{0} x_{t}=1 / 2, \quad \mathbf{D}_{0} x_{t}=1 / 4 .
$$

Отсюда, учитывая независимость слагаемых в сумме (9), получаем, что

$$
\mathbf{E}_{0} v_{m}^{(T)}\left(n_{i}\right)=\frac{T}{2 n_{i}}, \quad \mathbf{D}_{0} v_{m}^{(T)}\left(n_{i}\right)=\frac{T}{4 n_{i}} .
$$

Для вычисления ковариаций частот (9) воспользуемся равенством

$$
\operatorname{cov}_{0}\left(v_{m_{1}}^{(T)}\left(n_{i}\right), v_{m_{2}}^{(T)}\left(n_{j}\right)\right)=\sum_{t_{1} \in Z_{m_{1}}\left(n_{i}\right)} \sum_{t_{2} \in Z_{m_{2}}\left(n_{j}\right)} \operatorname{cov}_{0}\left(x_{t_{1}}, x_{t_{2}}\right)=\sum_{t \in Z_{m_{1}}\left(n_{i}\right) \cap Z_{m_{2}}\left(n_{j}\right)} \mathbf{D}_{0} x_{t} .
$$

Отсюда и из равенств (12) и (13) получаем, что

$$
\begin{array}{ll}
\operatorname{cov}_{0}\left(v_{m_{1}}^{(T)}\left(n_{i}\right), v_{m_{2}}^{(T)}\left(n_{j}\right)\right)=\frac{T}{4 n_{i} n_{j}}, & i \neq j, \\
\operatorname{cov}_{0}\left(v_{m_{1}}^{(T)}\left(n_{i}\right), v_{m_{2}}^{(T)}\left(n_{j}\right)\right)=0, & i=j, \quad m_{1} \neq m_{2} .
\end{array}
$$

Используя многомерную центральную предельную теорему и формулы (13) и (14), получим следующее утверждение.

Теорема 2. Если $n_{0}, n_{1}, \ldots, n_{r}$ постоянны, взаимно просты, $u L \rightarrow \infty$, то при гипотезе $H_{0}$ совместное распределение величин

$$
\theta_{m}^{(T)}\left(n_{i}\right)=\frac{v_{m}^{(T)}\left(n_{i}\right)-T /\left(2 n_{i}\right)}{\sqrt{T / n_{i}} / 2}, \quad m=1, \ldots, n_{i}, \quad i=0,1, \ldots, r
$$

сходится к распределению случайного вектора $\left\{\theta_{m}^{*}\left(n_{i}\right)\right\}$, имеющего нормальное распределение с нулевым вектором математических ожиданий и ковариационной матрицей $\left(\operatorname{cov}_{0}\left(v_{m_{1}}^{*}\left(n_{i}\right), v_{m_{2}}^{*}\left(n_{j}\right)\right)\right)$, где

$$
\begin{array}{lll}
\operatorname{cov}_{0}\left(v_{m_{1}}^{*}\left(n_{i}\right), v_{m_{2}}^{*}\left(n_{j}\right)\right)=1, & i=j, & m_{1}=m_{2}, \\
\operatorname{cov}_{0}\left(v_{m_{1}}^{*}\left(n_{i}\right), v_{m_{2}}^{*}\left(n_{j}\right)\right)=0, & i=j, & m_{1} \neq m_{2}, \\
\operatorname{cov}_{0}\left(v_{m_{1}}^{*}\left(n_{i}\right), v_{m_{2}}^{*}\left(n_{j}\right)\right)=\sqrt{n_{i} n_{j}}, & i \neq j .
\end{array}
$$

При гипотезе $H_{1}$ для моментов величин $x_{t}$, определенных равенством (4), нетрудно получить следующие выражения:

$$
\begin{aligned}
& \mathbf{E}_{1} x_{t}=1 / 2, \quad \mathbf{D}_{1} x_{t}=1 / 4, \\
& \operatorname{cov}_{1}\left(x_{t_{1}}, x_{t_{2}}\right)=0, \quad t_{1} \in M_{u}, \quad t_{2} \in M_{v}, \quad u \neq v, \\
& \operatorname{cov}_{1}\left(x_{t_{1}}, x_{t_{2}}\right)=p^{2} / 4, \quad t_{1}, t_{2} \in M_{u}, \quad t_{1} \neq t_{2} \text {. }
\end{aligned}
$$

Отсюда следует, что математическое ожидание частоты $v_{m}^{(T)}\left(n_{i}\right)$ при гипотезе $H_{1}$ совпадает с соответствующим математическим ожиданием при гипотезе $H_{0}$, то есть

$$
\mathbf{E}_{1} v_{m}^{(T)}\left(n_{i}\right)=\frac{T}{2 n_{i}}
$$


Частоты (9) представимы в виде

$$
v_{m}^{(T)}\left(n_{i}\right)=\sum_{u=1}^{n_{0}}\left(\sum_{t \in Z_{m}\left(n_{i}\right) \cap M_{u}} x_{t}\right), \quad v_{m}^{(T)}\left(n_{0}\right)=\sum_{t \in M_{m}} x_{t},
$$

где суммы в круглых скобках независимы.

Используя представление частот в виде (18), нетрудно получить следующее утверждение.

Теорема 3. Если последовательность $\left\{x_{t}\right\}$ определяется равенствами (3) u (4) и T имеет вид (11), то

$$
\begin{array}{rlrl}
\mathbf{D}_{1} v_{m}^{(T)}\left(n_{i}\right) & =\frac{1}{4}\left(\left(1-p^{2}\right) \frac{T}{n_{0}}+\frac{T^{2}}{n_{0}^{2}} p^{2}\right), & & \\
\mathbf{D}_{1} v_{m}^{(T)}\left(n_{i}\right) & =\frac{1}{4}\left(\left(1-p^{2}\right) \frac{T}{n_{i}}+\frac{T^{2}}{n_{0} n_{i}^{2}} p^{2}\right), & i \neq 0, & \\
\operatorname{cov}_{1}\left(v_{m_{1}}^{(T)}\left(n_{i}\right), v_{m_{2}}^{(T)}\left(n_{j}\right)\right) & =\frac{1}{4}\left(\left(1-p^{2}\right) \frac{T}{n_{i} n_{j}}+\frac{T^{2}}{n_{i} n_{j} n_{0}} p^{2}\right), \quad i \neq j, \quad i \neq 0, \quad j \neq 0, \\
\operatorname{cov}_{1}\left(v_{m_{1}}^{(T)}\left(n_{i}\right), v_{m_{2}}^{(T)}\left(n_{j}\right)\right) & =\frac{1}{4}\left(\left(1-p^{2}\right) \frac{T}{n_{0} n_{j}}+\frac{T^{2}}{n_{0}^{2} n_{j}} p^{2}\right), \quad i=0, \quad j \neq 0, \\
\operatorname{cov}_{1}\left(v_{m_{1}}^{(T)}\left(n_{i}\right), v_{m_{2}}^{(T)}\left(n_{j}\right)\right) & =\frac{1}{4}\left(p^{2} \frac{T^{2}}{n_{i}^{2} n_{0}^{2}}\right), \quad i=j \neq 0, \quad m_{1} \neq m_{2}, \\
\operatorname{cov}_{1}\left(v_{m_{1}}^{(T)}\left(n_{i}\right), v_{m_{2}}^{(T)}\left(n_{j}\right)\right) & =0, \quad i=j=0, \quad m_{1} \neq m_{2} .
\end{array}
$$

Докажем, например, формулу для случая $i \neq j, i \neq 0, j \neq 0$. Из представления $v_{m}^{(T)}\left(n_{i}\right)$ в виде (18), учитывая независимость сумм в круглых скобках, получаем равенство

$$
\operatorname{cov}_{1}\left(v_{m_{1}}\left(n_{i}\right), v_{m_{2}}\left(n_{j}\right)\right)=\sum_{u=1}^{n_{0}} \sum_{t_{1} \in Z_{m_{1}}\left(n_{i}\right) \cap M_{u}} \sum_{t_{2} \in Z_{m_{2}}\left(n_{j}\right) \cap M_{u}} \operatorname{cov}_{1}\left(x_{t_{1}}, x_{t_{2}}\right) .
$$

Сумму по $t_{1}, t_{2}$ представим в виде суммы двух слагаемых

$$
\Sigma_{1}(u)=\sum_{t \in Z^{(1)}} \mathbf{D}_{1} x_{t}, \quad \Sigma_{2}(u)=\sum_{\left(t_{1}, t_{2}\right) \in Z^{(2)}} \operatorname{cov}_{1}\left(x_{t_{1}}, x_{t_{2}}\right),
$$

где $Z^{(1)}=Z_{m_{1}}\left(n_{i}\right) \cap Z_{m_{2}}\left(n_{j}\right) \cap M_{u}$, а $Z^{(2)}$ состоит из пар $\left(t_{1}, t_{2}\right)$ с $t_{1} \neq t_{2}$. Нетрудно проверить, что

$$
\left|Z^{(1)}\right|=\frac{T}{n_{i} n_{j} n_{0}}, \quad\left|Z^{(2)}\right|=\frac{T}{n_{i} n_{0}} \frac{T}{n_{j} n_{0}}-\frac{T}{n_{i} n_{j} n_{0}} .
$$

Из (16), (18) и (20) получаем первую формулу для ковариаций. Остальные формулы доказываются аналогично.

По гипотезе $H_{1}$, дисперсии частот при $T \rightarrow \infty$ имеют порядок $T^{2}$. Дисперсии частот по условному распределению при фиксированном векторе $y\left(n_{0}\right)=\left(y_{1}, y_{2}, \ldots, y_{n_{0}}\right)$ имеют обычный порядок $T$. При $t \in M_{m}$

$$
\mathbf{P}_{1}\left\{x_{t}=1 \mid y_{m}=1\right\}=\frac{1+p}{2}, \quad \mathbf{P}_{1}\left\{x_{t}=1 \mid y_{m}=0\right\}=\frac{1-p}{2} .
$$


При условии $y_{m}$ величины $x_{t}, t \in M_{m}$ независимы и

$$
\begin{array}{r}
\mathbf{E}_{1}\left(v_{m}^{(T)}\left(n_{0}\right) \mid y_{m}=1\right)=\frac{T(1+p)}{2 n_{0}}, \\
\mathbf{E}_{1}\left(v_{m}^{(T)}\left(n_{0}\right) \mid y_{m}=0\right)=\frac{T(1-p)}{2 n_{0}}, \\
\mathbf{D}_{1}\left(v_{m}^{(T)}\left(n_{0}\right) \mid y_{m}=1\right)=\mathbf{D}_{1}\left(v_{m}^{(T)}\left(n_{0}\right) \mid y_{m}=0\right)=\frac{T\left(1-p^{2}\right)}{4 n_{0}} .
\end{array}
$$

Таким образом, при $T \rightarrow \infty$

$$
\begin{aligned}
& \mathbf{P}_{1}\left\{\frac{v_{m}^{(T)}\left(n_{0}\right)-T(1+p) /\left(2 n_{0}\right)}{(1 / 2) \sqrt{T\left(1-p^{2}\right) / n_{0}}}<x \mid y_{m}=1\right\} \rightarrow \Phi(x), \\
& \mathbf{P}_{1}\left\{\frac{v_{m}^{(T)}\left(n_{0}\right)-T(1-p) /\left(2 n_{0}\right)}{(1 / 2) \sqrt{T\left(1-p^{2}\right) / n_{0}}}<x \mid y_{m}=0\right\} \rightarrow \Phi(x),
\end{aligned}
$$

где $\Phi(x)$ - функция нормального распределения с параметрами $(0,1)$. Таким образом, распределение $v_{m}^{(T)}\left(n_{0}\right)$ сосредоточено с равными вероятностями вокруг удаляющихся друг от друга точек $T(1+p) /\left(2 n_{0}\right)$ и $T(1-p) /\left(2 n_{0}\right)$. Распределение $v_{m}\left(n_{i}\right)$ при $i \neq 0$ является смесью $n_{0}+1$ распределений, зависящих от суммы $\mu_{n_{0}}=y_{1}+\ldots+y_{n_{0}}$.

\section{4. Моменты и распределения статистик}

Математические ожидания частот $v_{m}^{(T)}\left(n_{i}\right)$ при обеих гипотезах одинаковы. Статистики (7), зависящие от частот $v_{m}^{(T)}\left(n_{i}\right)$, можно заменить статистиками, зависящими от центрированных частот и отличающимися от частот (7) постоянными слагаемыми:

$$
\xi_{n}^{*}(T)=\sum_{m=1}^{n} \ln \left(A^{\hat{\theta}_{m}^{(T)}(n)}+A^{-\hat{\theta}_{m}^{(T)}(n)}\right), \quad n=n_{0}, n_{1}, \ldots, n_{r},
$$

где

$$
\hat{\theta}_{m}^{(T)}(n)=v_{m}^{(T)}(n)-\frac{T}{2 n}, \quad A=\frac{1+p}{1-p} .
$$

Теорема 4. Если $n_{0}, n_{1}, \ldots, n_{r}$ постоянны, взаимно просты, $T=n_{0} n_{1} \ldots n_{r} L u L \rightarrow \infty$, то при гипотезе $H_{0}$ распределение вектора $(2 / \sqrt{A} \ln A)\left(\sqrt{n_{0}} \xi_{n_{0}}^{*}(T), \ldots, \sqrt{n_{r}} \xi_{n_{r}}^{*}(T)\right)$ сходится к совместному распределению величин $\sum_{m=1}^{n_{i}}\left|\theta_{m}^{*}\left(n_{i}\right)\right|, i=0,1, \ldots, r$, где величинь $\theta_{m}^{*}\left(n_{i}\right)$ определены в формулировке теоремы 2.

Доказательство. Представим статистики (23) в виде

$$
\xi_{n}^{*}(T)=\sum_{m=1}^{n}\left(\ln A^{\left|\theta_{m}^{(T)}(n)\right| \sqrt{T / n} / 2}+\Delta_{m}^{(T)}(n)\right),
$$

где

$$
\begin{aligned}
\theta_{m}^{(T)}(n) & =2 \hat{\theta}_{m}^{(T)}(n) / \sqrt{T / n} \\
\Delta_{m}^{(T)}(n) & =\ln \left(A^{\left(\theta_{m}^{(T)}(n)-\left|\theta_{m}^{(T)}(n)\right|\right) \sqrt{T / n} / 2}+A^{-\left(\theta_{m}^{(T)}(n)-\left|\theta_{m}^{(T)}(n)\right|\right) \sqrt{T / n} / 2}\right) .
\end{aligned}
$$


Положим $\gamma_{m}=1$, если $\theta_{m}^{(T)}(n)>0$, и $\gamma_{m}=0$, если $\theta_{m}^{(T)} Z(n)<0$.

Второе равенство (26) можно записать в виде

$$
\Delta_{m}^{(T)}(n)=\gamma_{m} \ln \left(1+A^{-\theta_{m}^{(T)}(n) \sqrt{T / n}}\right)+\left(1-\gamma_{m}\right) \ln \left(1+A^{\theta_{m}^{(T)}(n) \sqrt{T / n}}\right) .
$$

Отсюда

$$
\Delta_{m}^{(T)}(n)=\ln \left(1+A^{-\left|\theta_{m}^{(T)}(n)\right| \sqrt{T / n}}\right)
$$

Из теоремы 2 следует, что величины $\Delta_{m}^{(T)}(n)$ при $L \rightarrow \infty$ сходятся по вероятности к нулю. Таким образом, предельные распределения $\xi_{n_{i}}^{*}(T)$ и $\sum_{m=1}^{n_{i}}\left(\frac{1}{2} \sqrt{T / n_{i}} \ln A\right)\left|\theta_{m}^{(T)}\left(n_{i}\right)\right|$, $i=0,1, \ldots, r$, совпадают. Теорема доказана.

Рассмотрим более простые (по сравнению с (23)) статистики, для которых можно получать простые явные выражения для моментов. Разлагая слагаемые в (23) в ряд Тейлора по степеням $\hat{\theta}_{n}^{(T)}(n)$, получим, что

$$
\ln \left(A^{\hat{\theta}_{m}^{(T)}(n)}+A^{-\hat{\theta}_{m}^{(T)}(n)}\right)=\ln 2+\frac{1}{2}\left(\hat{\theta}_{m}^{(T)}(n)\right)^{2}(\ln A)^{2}+\ldots
$$

Определим статистики $\eta_{n_{i}}(T)$ равенствами

$$
\eta_{n}(T)=\sum_{m=1}^{n}\left(\hat{\theta}_{m}^{(T)}(n)\right)^{2}, \quad n=n_{0}, n_{1}, \ldots, n_{r},
$$

где $\hat{\theta}_{m}^{(T)}(n)$ определены равенствами (24).

При гипотезах $H_{0}$ и $H_{1}$ из формул (13), (17) и (27) следует, что

$$
\mathbf{E}_{l} \eta_{n}(T)=\sum_{m=1}^{n} \mathbf{D}_{l} v_{m}(n), \quad l=0,1 .
$$

Отсюда, из формул (13) и теоремы 3 получаем следующие два утверждения.

Следствие 1. При гипотезе $H_{0} u T=n_{0} n_{1} \ldots n_{r} L$

$$
\mathbf{E}_{0} \eta_{n}(T)=T / 4, \quad n=n_{0} n_{1} \ldots n_{r} .
$$

Следствие 2. Если числа $n_{0}, n_{1}, \ldots, n_{r}$ взаимно просты, то при гипотезе $H_{1} u$ $T=n_{0} n_{1} \ldots n_{r} L$

$$
\begin{aligned}
& \mathbf{E}_{1} \eta_{n_{0}}(T)=\frac{1}{4}\left(p^{2} T^{2} / n_{0}+\left(1-p^{2}\right) T\right), \\
& \mathbf{E}_{1} \eta_{n_{0}}(T)=\frac{1}{4}\left(p^{2} T^{2} /\left(n n_{0}\right)+\left(1-p^{2}\right) T\right), \quad n=n_{0}, \ldots, n_{r} .
\end{aligned}
$$

Из полученных формул следует, что

$$
\begin{array}{ll}
\lim _{T \rightarrow \infty} \frac{4 n_{0}}{p^{2} T^{2}} \mathbf{E}_{1} \eta_{n}(T)=1, & n=n_{0}, \\
\lim _{T \rightarrow \infty} \frac{4 n_{0}}{p^{2} T^{2}} \mathbf{E}_{1} \eta_{n}(T)=\frac{1}{n_{i}}, & n=n_{1}, \ldots, n_{r},
\end{array}
$$

Если получить несколько реализаций $T$ первых членов последовательности $\left\{x_{t}\right\}$, то можно оценить $\mathbf{E}_{1} \eta_{n}(T)$ и найти $n_{0}$. Возможность определения $n_{0}$ по одной реализации рассматривается в следующем параграфе. 


\section{5. Моменты статистик относительно условных распределений}

При любом $T>n_{0}$ случайные величины $x_{t}$, определенные равенствами (4), зависят от одних и тех же величин $y\left(n_{0}\right)=\left(y_{1}, \ldots, y_{n_{0}}\right)$. Найдем моменты частот и статистик при заданных значениях $y\left(n_{0}\right)$.

Из формул (21) и (22) следует, что

$$
\mathbf{P}_{1}\left\{x_{t}=1 \mid y_{m}\right\}=\frac{1}{2}\left(1-\left(1-2 y_{m}\right) p\right), \quad t \in M_{m} .
$$

Величины $\hat{\theta}_{m}^{(T)}(n)$ представим в виде

$$
\begin{aligned}
\hat{\theta}_{m}^{(T)}\left(n_{0}\right) & =\sum_{t \in M_{n}}\left(x_{t}-1 / 2\right), \\
\hat{\theta}_{m}^{(T)}(n) & =\sum_{u=1}^{n_{0}} \sum_{t \in Z_{m}(n) \cap M_{u}}\left(x_{t}-1 / 2\right), \quad n=n_{1}, \ldots, n_{r} .
\end{aligned}
$$

Из равенства (28) при $t \in M_{u}$ получаем, что $\left.\mathbf{E}_{1}\left(x_{t}-1 / 2\right) \mid y_{m}\right)=p\left(y_{m}-1 / 2\right)$. Отсюда следует, что по условному распределению с фиксированным $y\left(n_{0}\right)$ средние арифметические одинаково распределенных случайных величин $\left(n_{i} n_{0} / T\right) \sum_{t \in Z_{m}\left(n_{i}\right) \cap M_{u}}\left(x_{t}-1 / 2\right)$, $\left(n_{0} / T\right) \sum_{t \in M_{m}}\left(x_{t}-1 / 2\right)$ при $T \rightarrow \infty$ сходится по вероятности к величинам $p\left(y_{u}-1 / 2\right)$ и $p\left(y_{m}-1 / 2\right)$ соответственно. Используя (29), получаем, что величины $(1 / T) \hat{\theta}_{m}^{(T)}\left(n_{i}\right)$, $(1 / T) \hat{\theta}_{m}^{(T)}\left(n_{0}\right)$ сходятся по вероятности к $\left(p /\left(n_{i} n_{0}\right)\right)\left(\mu_{n_{0}}-n_{0} / 2\right),\left(p / n_{0}\right)\left(y_{m}-1 / 2\right)$, $\mu_{n_{0}}=y_{1}+\ldots+y_{n_{0}}$, а также сходятся случайные величины $\eta_{n_{i}}(T) / T^{2}$ и $\eta_{n_{0}}(T) / T^{2}$. Таким образом, доказано следующее утверждение.

Теорема 5. Совместное условное распределение случайных величин $\eta_{n_{i}}(T) / T^{2}$, $\eta_{0}(T) / T^{2}, i=1, \ldots, r$, с фиксированным $y\left(n_{0}\right)=\left(y_{1}, \ldots, y_{n_{0}}\right)$ при $T \rightarrow \infty$ слабо сходится к вырожденному распределению, сосредоточенному в точке

$$
\frac{p^{2}}{4 n_{0}}, \quad \frac{p^{2}}{n_{i} n_{0}^{2}}\left(\mu_{n_{0}}-\frac{n_{0}}{2}\right)^{2}, \quad i=1, \ldots, r .
$$

\section{6. Примеры}

Рассмотрим статистики

$$
\zeta_{n}^{(1)}(T)=\xi_{n}^{*}(T), \quad \zeta_{n}^{(2)}(T)=\eta_{n}(T), \quad \zeta_{m}^{(3)}(T)=Y_{n}^{*}(T),
$$

где $\zeta_{n}^{(1)}(T), \zeta_{n}^{(2)}(T)$ определены формулами (23), (24) и (27), а

$$
Y_{n}^{*}(T)=\frac{T}{2}|X(2 \pi / n)|^{2}, \quad X(\omega)=\frac{2}{T} \sum_{t=1}^{T}\left(2 x_{t}-1\right) \exp \{i \omega t\} .
$$

Статистики (31) введены вместо статистик (2) для удобства сравнения со статистиками $\zeta_{n}^{(k)}(T)$. Статистики (2) зависят от частот $\omega_{j}=2 \pi j / T$ и занумерованы числами $j$, а статистики (32) занумерованы периодами $n$, соответствующими частотам $\omega_{j}$ с $j=T / n$. 
Таблица 1.

\begin{tabular}{llllllll}
\hline$k$ & $\xi_{n}^{(k)}(T)$ & $\widehat{E}_{12}(k)$ & $\widehat{E}_{13}(k)$ & $\widehat{E}_{14}(k)$ & $\hat{p}_{12}(k)$ & $\hat{p}_{13}(k)$ & $\hat{p}_{14}(k)$ \\
\hline 1 & $\xi_{n}^{*}(T)$ & 22,1 & 46,1 & 23,5 & 0 & 0,993 & 0,002 \\
2 & $\eta_{n}(T)$ & 113 & 413 & 105 & 0 & 0,983 & 0 \\
3 & $Y_{n}^{*}(T)$ & 2,01 & 8,59 & 1,81 & 0,049 & 0,590 & 0,049 \\
\hline
\end{tabular}

Таблица 2.

\begin{tabular}{llllllll}
\hline$k$ & $\zeta_{n}^{(k)}(T)$ & $\widehat{E}_{12}(k)$ & $\widehat{E}_{13}(k)$ & $\widehat{E}_{14}(k)$ & $\hat{p}_{12}(k)$ & $\hat{p}_{13}(k)$ & $\hat{p}_{14}(k)$ \\
\hline 1 & $\xi_{n}^{*}(T)$ & 12,6 & 16,1 & 14,0 & 0,002 & 0,484 & 0,054 \\
2 & $\eta_{n}(T)$ & 99,3 & 179 & 96,6 & 0,037 & 0,620 & 0,035 \\
3 & $Y_{n}^{*}(T)$ & 1,96 & 3,70 & 2,00 & 0,083 & 0,270 & 0,095 \\
\hline
\end{tabular}

Пусть $n_{0}=13$ и возможное множество периодов $\left\{n_{0}, n_{1}, \ldots, n_{8}\right\}=\{8,9, \ldots, 16\}$, $T=390$. Оценку $\hat{n}_{0}(k)$ периода $n$, найденную по последовательности (1) с использованием статистики $\zeta_{n}^{(k)}(T)$, определим равенством

$$
\zeta_{\hat{n}_{0}(k)}^{(k)}(T)=\max _{8 \leqslant n \leqslant 16} \zeta_{n}^{(k)}(T),
$$

Для иллюстрации свойств статистик (31) было смоделировано по 1000 реализаций $T=390$ первых членов последовательности (1) при гипотезе $H_{1}$ с $p=1 / 3$ и $p=1 / 6$. Получены оценки $\widehat{E}_{n}(k), \hat{p}_{n}(k)$ величин

$$
\mathbf{E} \zeta_{n}^{(k)}(T)=E_{n}(k), \quad p_{n}(k)=\mathbf{P}\left\{\hat{n}_{0}(k)=n\right\}, \quad k=1,2,3, \quad 12 \leqslant n \leqslant 14 .
$$

Эти оценки приведены в табл. 1 для $p=1 / 3$ и табл. 2 для $p=1 / 6$.

\section{Список литературы}

1. Бриллинджер Д., Временные ряды. Мир, Москва, 1980.

2. Харин Ю. С., Берник В. И., Матвеев Г. В., Агиевич С. В., Математические и компьютерные основы криптографии. Новое знание, Минск, 2003.

Статья поступила 14.09.2006. 